\title{
CHARITÉ
}

\section{Rechtskreisübergreifende Kooperation in einem Gemeindepsychiatrischen Zentrum}

\section{Eine qualitative Studie über die Erfahrungen der Stakeholder}

Julian Schwarz, Barbara Stöckigt, Anne Berghöfer, Sebastian von Peter, Burkhart Brückner

\section{Document type}

Postprint (accepted version)

This version is available at

https://doi.org/10.17169/refubium-32721

\section{Citation details}

Schwarz J, Stöckigt B, Berghöfer A, von Peter S, Brückner B. Rechtskreisübergreifende Kooperation in einem Gemeindepsychiatrischen Zentrum. Psychiatrische Praxis. Georg Thieme Verlag KG; 2018. p. 200-205. DOI: 10.1055/a-0800-3783

\section{Terms of use}

All rights reserved. This document is intended solely for personal, non-commercial use. 
Titel

Rechtskreisübergreifende Kooperation in einem

Gemeindepsychiatrischen Zentrum

Eine qualitative Studie über die Erfahrungen der Stakeholder

Interagency cooperation in a

Community Mental Health Centre

A Qualitative Study about the Stakeholders' Experiences

\section{Autoren}

Julian Schwarz ${ }^{1}$, Barbara Stöckigt ${ }^{2}$, Anne Berghöfer ${ }^{2}$, Sebastian von Peter ${ }^{1}$, Burkhart

Brückner $^{3}$

1) Hochschulklinik für Psychiatrie und Psychotherapie der Medizinischen Hochschule Brandenburg, Immanuel Klinik Rüdersdorf

Dr. med. Julian Schwarz

Seebad 82/83

15562 Rüdersdorf bei Berlin

julian.schwarz@mhb-fontane.de

2) Institut für Sozialmedizin, Epidemiologie und Gesundheitsökonomie, Charité Universitätsmedizin Berlin, Berlin

3) Fachbereich Sozialwesen, Hochschule Niederrhein University of Applied Sciences, Mönchengladbach 


\section{Schlüsselwörter}

Rechtskreisübergreifende Kooperation, sektorenübergreifend, Eingliederungshilfe, Psychosoziale Beratung, qualitative Studie

\section{Keywords}

interagency cooperation, intersectoral, social integration services, psychosocial counselling, qualitative study

\section{ZUSAMMENFASSUNG}

- Ziel Untersucht werden die Kooperationsbedingungen der beteiligten Akteure in einem Modellprojekt zur Koordination von SGB V und XII Leistungen.

- Methode Interviews mit gemeindepsychiatrischen Akteuren aus der Modellregion und qualitativinhaltsanalytischer Auswertung.

- Ergebnisse Ähnliche Steuerungsziele und Vergütungsmodelle sind Voraussetzungen rechtskreisübergreifender Zusammenarbeit. Gemeindeferne Versorgung sowie (infra-) strukturelle Defizite wirken sich hinderlich aus.

- Schlussfolgerungen Die Implementierung sektorenübergreifender Finanzierung für Behandlungs- als auch Eingliederungsleistungen sind ein erster Schritt in Richtung rechtskreisübergreifender Kooperation.

\section{ABSTRACT}

- Objective This study explores the conditions for the stakeholders' cooperation in an integrative care model for people with psychosocial problems.

- Methods Expert interviews on various community mental health care providers were led and content analyzed.

- Results Joint objectives and conceptually comparable financing models were found to be essential for cooperation across mental health and social integration services.

Conclusions Implementation of intersectoral financing for both clinical and social integration services can promote interagency cooperation. 


\section{Einleitung}

Im letzten Jahrzehnt wurden erhebliche Bemühungen unternommen, um eine gemeindepsychiatrische Basisversorgung zu ermöglichen [1-3]. Bisher ungelöst bleibt, wie die Leistungen unterschiedlicher Sozialgesetzbücher übergreifend erbracht werden können [4]. Auch gegenwärtige Reformen wie das PsychVVG oder das Teilhabegesetz tragen nur begrenzt zur Lösung der Problematik bei: Sie stärken sektorenübergreifende Behandlungs- und Unterstützungsformen, lassen jedoch den Aspekt der Integration von Leistungen unterschiedlicher Sozialgesetzbücher außer Acht [5].

Dass dies kein Spezifikum des deutschen Gesundheits- und Sozialsystems ist, zeigt die internationale Literatur [6-8]. V.a. in Skandinavien und Australien wurde untersucht, wie psychiatrische und komplementäre Leistungserbringer enger kooperieren und ihre Hilfen integrativ erbringen können [9-14]. Empfohlen wird insbesondere die Implementierung konkreter Kooperationsmaßnahmen [6,7]. Auch aus Deutschland liegen dazu einzelne Forschungs- und Erfahrungsberichte vor [15-17].

Ebenfalls räumt das Funktionale Basismodell für die gemeindepsychiatrische Versorgung schwer psychisch kranker Menschen dem rechtskreisübergreifenden Aspekt einen erheblichen Stellenwert ein [18]. Dieses Modell definiert Struktur- und Leistungsmerkmale künftiger psychosozialer Versorgung. Ausgehend von einer zentralen Steuerungs- und Beratungsfunktion, die sämtliche psychosoziale Hilfen koordiniert, bilden mobile multiprofessionelle Teams die operative Grundlage des Modells.

\section{Studienregion}

Der ca. 130.000 Einwohner große Landkreis Dithmarschen eignet sich für eine empirische Untersuchung der Bedingungen für eine solche Kooperation, da hier gezielt Maßnahmen für eine rechtskreis- und sektorenübergreifende Zusammenarbeit implementiert wurden. Hierzu gehören die

1. Einführung des regionalen Psychiatriebudgets (= RPB) am Westküstenklinikum Heide im Jahr 2008 [19,20]. Die Klinik erhält für jeden Patienten eine Kopfpauschale pro Jahr (Capitation-Model), um die psychiatrische Akutversorgung sicherzustellen. Die Budgetfinanzierung ermöglicht $u$. a. eine settingflexible und verstärkt ambulante Behandlung unter Berücksichtigung personeller Kontinuität [21].

2. Erprobung eines Abrechnungsbudgets für einen größeren Anbieter von Eingliederungsleistungen im Bereich Teilhabe Wohnen. Analog zum RPB stellt dies eine gleichbleibende Vergütung unabhängig von der in Anspruch genommenen Leistungsform sicher. Ziel ist es, Sozialunternehmen dabei zu unterstützen stationäre Wohnformen sukzessive in (intensive) ambulante Hilfen zu überführen, ohne dass dies zu Mindererlösen führt.

3. Einrichtung eines gemeindepsychiatrischen Zentrums (= GPZ) im Jahr 2014, das über die Funktionsbereiche Beratung und Koordination von Leistungen für Behandlung und Teilhabe (SGB V und XII) verfügt. Das Ziel, Nutzer frühzeitig in bedarfsgerechte und ambulante Leistungen einzusteuern, wird in einem rechtskreisübergreifend besetzten 
Case-Management realisiert. Abb. 1 zeigt die Einbettung des GPZ in die Strukturen der psychosozialen Versorgung in Dithmarschen sowie die strukturellen und prozessualen Besonderheiten der Fallsteuerung.

\section{Studiendesign und Fragestellung}

Die Untersuchung ist Bestandteil einer dreiteiligen Studie zur Evaluation des GPZ. Studienteil I und || analysierten die Kostenentwicklung und Kosteneffektivität von Leistungen der Eingliederungshilfe (= EGH) seit Implementierung. Ökonomische Evaluationen zeigen, dass die Beratungs- und Koordinationsarbeit des GPZ kosteneffektiv ist: Bei einem Anstieg der Lebensqualität im Zeitraum nach Inanspruchnahme des GPZ sanken die Ausgaben insbesondere im stationären Bereich [22,23]. Studienteil III explorierte die Sichtweisen der Stakeholder (zur Nutzerperspektive vgl. [24]). Der vorliegende Artikel untersucht die Kooperationsbedingungen der professionellen Akteure im GPZ:

Welche Bedingungen fördern die Zusammenarbeit zwischen den Sektoren- und Rechtskreisen? Was sind Voraussetzungen, Hindernisse und Herausforderungen der Kooperation?

\section{SEKTORENÜBERGREIFEND}

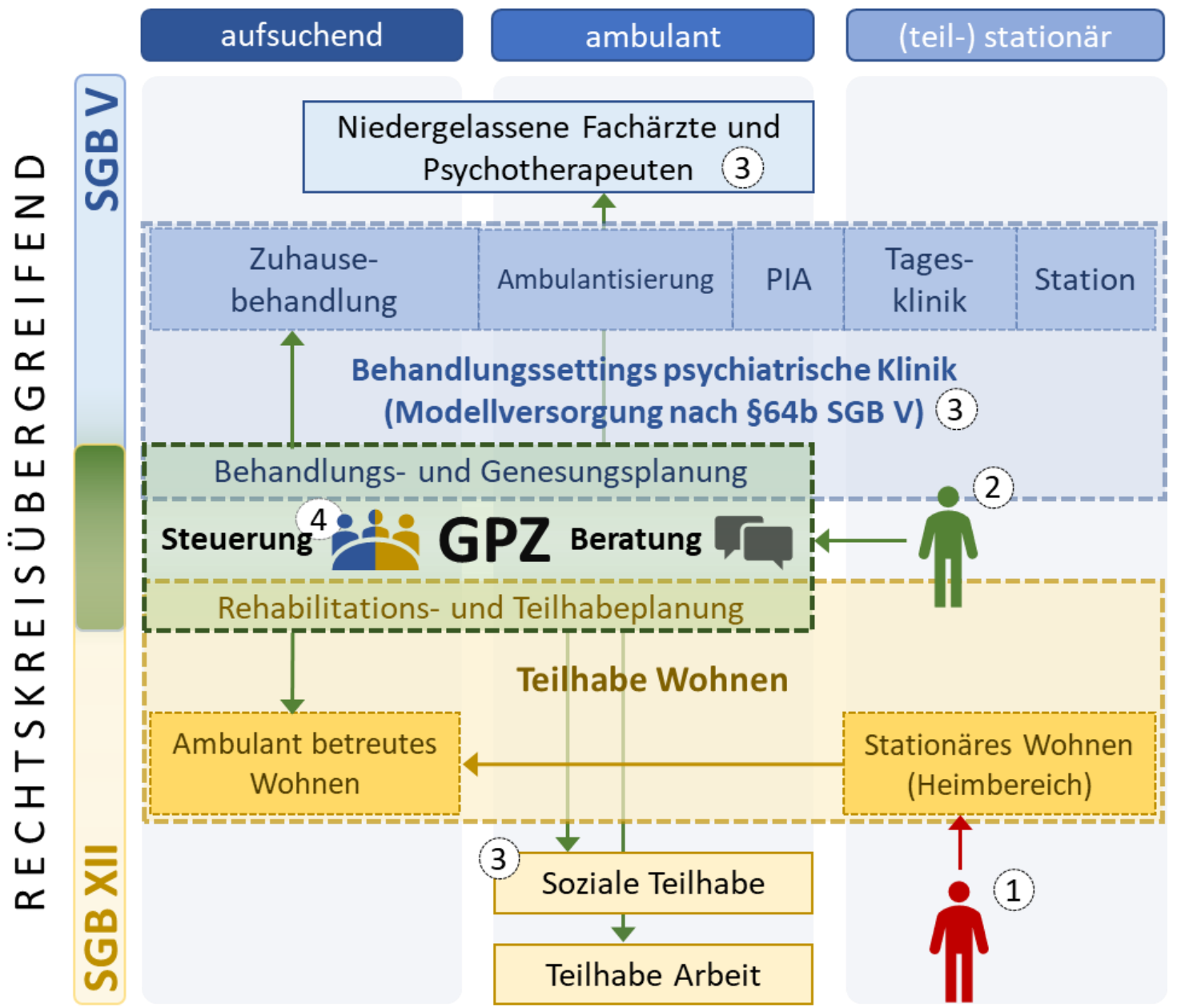

Abb. 1 Einbettung des GPZ in die Strukturen der psychosozialen Versorgung im Landkreis Dithmarschen. Adaptiert nach Steinhart und Wienberg [18]. 
Begleittext Abb. 1: Beschreibung der strukturellen und prozessualen Besonderheiten der rechtskreisübergreifenden Fallsteuerung im GPZ.

1: Vor Bestehen des GPZ wurden (teil-) stationäre EGH-Leistungen ungesteuert angeboten. „Neufälle“ mit komplexen Versorgungsbedarfen wurden je nach Belegungssituation in stationäre Wohnformen aufgenommen.

2: Kontaktaufnahme mit dem GPZ. Es werden der Hilfebedarf eingeschätzt, supportive Gespräche angeboten und zu medizinischen (SGB V) sowie Teilhabeleistungen (SGB XII) beraten, ggf. auch aufsuchend.

3: Bei größerem Unterstützungsbedarf wird qualifiziert weitervermittelt, etwa an die psychiatrische Klinik, ambulante Therapeuten, niedergelassene Fachärzte oder komplementäre Angebote zur sozialen Teilhabe (z.B. Kontakt- und Begegnungsstätten).

4: Patienten mit komplexen Hilfebedarfen werden in der sogenannten „GPZ-Auftragsrunde“ (= Case-Management) beraten, an der Fallmanager der EGH, Mitarbeiter des GPZ sowie Bezugstherapeuten der Klinik teilnehmen, u.a. auch ein Facharzt für Psychiatrie. Geleitet wird die Runde vom GPZ-Koordinator, dessen Stelle von der Klinik und der EGH gemeinsam finanziert wird. Die Koordinationstätigkeit in der GPZ-Auftragsrunde findet anbieterneutral statt, um eine verstärkte Orientierung an den Bedürfnissen der Nutzer und nicht an den Partikularinteressen der Leistungserbringer zu ermöglichen. Damit verbunden ist das langfristige Ziel, stationäre Leistungen zu limitieren und ambulante Angebote auszubauen.

\section{Methode}

Aufgrund der schmalen Studienlage zur Forschungsthematik und da es sich um eine neuartige Versorgungsstruktur handelt, wurde ein exploratives, qualitatives Vorgehen gewählt. Um die Perspektiven der Teilnehmenden zu evaluieren und „eine möglichst unvoreingenommene Abbildung individueller Handlungen sowie subjektiver Wahrnehmungen und Verarbeitungsweisen gesellschaftlicher Realität“ zu ermöglichen, wurden problemzentrierte Experteninterviews gewählt [25]. Die Studie wurde von der Ethikkommission der Ärztekammer Schleswig-Holstein bewilligt (Az. 131/14).

\section{Sampling und Datenerhebung}

Es erfolgte ein Convenience Sampling, wobei alle Akteure, die unmittelbar in die Arbeit im GPZ involviert sind, ausgewählt wurden. Die Interviews wurden vom Erstautor geführt. Es wurden die Voraussetzungen, Bedingungen und Hürden von SGB-übergreifender Kooperation gezielt exploriert. Die Interviews wurden digital aufgezeichnet, transkribiert und pseudonymisiert.

\section{Datenanalyse}

Die Auswertung erfolgte mittels zusammenfassender qualitativer Inhaltsanalyse nach Mayring [26]. Die Erhebung und Analyse fanden abwechselnd statt, bis keine neuen Erkenntnisse mehr erzielt wurden, also „theoretische Sättigung“ eintrat [27]. In einem alternierend deduktivinduktiven Prozess entstand ein System aus Haupt- und Unterkategorien. Um substanzielle Gütekriterien qualitativer Forschung zu berücksichtigen, wurden die Transkripte konsensuell kodiert. Der Fortgang von Analyse und Auswertung wurde regelmäßig im Autorenteam diskutiert.

\section{Ergebnisse}

Insgesamt wurden 9 Interviews geführt (5 Frauen, 4 Männer). Tab. 1 zeigt die Zusammensetzung der Stichprobe. Die durchschnittliche Interviewdauer betrug 48 Minuten. Das zugehörige Kategoriensystem finden Sie im Online-Material (Abb. 2). 
Tab. 1 Zusammensetzung der Stichprobe $(n=9)$

\begin{tabular}{|l|l|l|l|}
\hline Institution, Berufsgruppe bzw. Funktion & Kürzel & $\begin{array}{l}\text { Anzahl der } \\
\text { Teilnehmer }\end{array}$ & $\begin{array}{l}\text { davon mit } \\
\text { Leitungsfunktion }\end{array}$ \\
\hline $\begin{array}{l}\text { Psychiatrische Klinik } \\
\text { (Arzt, Psychologin, Pflegefachperson) }\end{array}$ & Klinik & 3 & 2 \\
\hline $\begin{array}{l}\text { Gemeindepsychiatrisches Zentrum } \\
\text { (Pflegefachpersonen) }\end{array}$ & GPZ & 2 & 1 \\
\hline $\begin{array}{l}\text { Kostenträger Eingliederungshilfe } \\
\text { (Fachdienstleiterin und Case-Manager) }\end{array}$ & EGH & 3 & 1 \\
\hline $\begin{array}{l}\text { Leistungserbringer Eingliederungshilfe } \\
\text { (Leiterin einer stationären Heimeinrichtung) }\end{array}$ & Heim & 1 & 1 \\
\hline
\end{tabular}

\section{Voraussetzungen}

Alle Befragten sahen gemeinsame Versorgungsziele als Grundbedingung der Zusammenarbeit an. Dies sei nicht nur aus institutioneller Perspektive bedeutsam, sondern fördere auch eine „gemeinsame kooperative Haltung“ zwischen den Mitarbeitern der Einrichtungen:

„Wir haben festgestellt, dass wir gleiche Interessen haben. Wir haben diesen Prozess der Ambulantisierung durchgemacht, möchten also gar nicht mehr den Patienten so viel stationär haben." (Klinik_4)

Ähnliche Vergütungsmodelle (hier: Budgetfinanzierung) wurden von allen Akteursgruppen als wichtige Voraussetzung eingestuft. Der Budgetgedanke ermögliche es u.a. hohe Betreuungsintensitäten in niederschwelligen Settings zu erbringen:

„Das System ist nicht flexibel genug gewesen, es muss nicht diese Treppe sein: vollstationär viel Hilfe, teilstationär weniger. Unser Anspruch ist jetzt, auch viel Hilfe in die Wohnungen zu bringen." (Heim_9)

\section{Fördernde Aspekte}

Die Implementierung des SGB-übergreifenden Case-Managements unter Leitung eines Fallmanagers wurde von allen Akteuren als besonders kooperationsfördernd angesehen und ermöglicht eine passgenaue Abstimmung von Komplexleistungen:

„Es geht darum, zu einem Ergebnis zu kommen, deswegen haben wir auch einmal die Woche [das Case-Management] für Klienten, deren Leistungen nicht geklärt sind. Und das Ergebnis ist immer: Wer, macht's denn jetzt? Das wir uns nicht wochenlang darüber streiten müssen, während es der Patientin schlechter geht - und einer muss halt in den sauren Apfel beißen." (GPZ_8)

Förderlich für die Kooperation seien Regelungen zum strukturierten Wissens- und Erfahrungsaustausch. Durch den Einbezug der Klinik bei Ambulantisierungsprozessen seitens Leistungserbringer der $\mathrm{EGH}$, habe ein gezielter Erfahrungstransfer stattgefunden:

„Die Kooperation findet seit ein paar Monaten sehr intensiv statt, [...] d.h. wenn der EGH-Anbieter sagt, wir wollen ambulantisieren, dann ist in jeder Arbeitsgruppe ein Mitarbeiter der Klinik dabei und kann nochmal die eigenen Erfahrungen aus dem Regionalbudget zum Besten geben." (GPZ_8) 
„Das Verständnis füreinander ist durch Fortbildungen gestärkt worden und auch der konstruktive Austausch über Ambulantisierungsprozesse hat stattgefunden. " (GPZ_7)

Aus Sicht der Befragten hatte der Einsatz von GPZ-Mitarbeitern in Personalunion zahlreiche Synergieeffekte. Einerseits würden Kommunikationsbarrieren reduziert und bei Konflikten könne schneller interveniert werden. Anderseits sei die Einnahme der Doppelrolle, die sich aus der Anstellung bei Klinik und EGH ergebe, oft nicht einfach. Für diese koordinative Funktion seien insbesondere Mitarbeiter mit langjähriger Kenntnis der regionalen „Psychiatrie-Szene“ geeignet:

"Und dann haben wir einfach Erfahrungen mit geteiltem Personal gesammelt und siehe da-das funktioniert. Also es gibt sehr sehr viel weniger Konflikte." (Klinik_4)

"Aber deshalb ist das ja gerade ganz wichtig, dass man auch die Leute kennt. Man muss einfach wissen, wo ich wen hin verweisen kann." (GPZ_7)

Alle Akteure stimmten darin überein, dass die gemeinsam verbrachte Zeit die Kooperation gestärkt und Kommunikationsprozesse gefördert habe:

„[Die Zusammenarbeit hat sich] einfach dadurch entwickelt, dass wir mehr Zeit miteinander verbracht haben, dass wir uns kennengelernt haben, dass die Klinikmitarbeiter auch gewusst haben, so, wer ist der Fachdienst EGH, was machen die überhaupt? Und andersherum genauso; man musste die Ärzte kennenlernen." (EGH_3)

„Verändert hat sich der schnellere Zugriff der Mitarbeiter untereinander. [...] Früher war es so, dass man mehrere Wochen hat warten müssen auf einen Termin beim Fachdienst EGH. Jetzt reicht ein Anruf [...] - man vernetzt sich kooperativer." (GPZ_8)

\section{Hindernisse und Herausforderungen}

Als eine Hürde der Zusammenarbeit benannten Klinik und EGH die Versorgung von Klienten aus entfernten Regionen. Für deren Hilfeplanung sei die EGH des Herkunftskreises zuständig. Letztere sei jedoch mit den Hilfsangeboten der Studienregion nicht vertraut, was die Hilfeplanung erschwere. Klinikmitarbeiter gaben an, dass es sich zumeist um Patienten mit komplexen Unterstützungsbedarfen (sog. „Systemsprenger“) handle, die häufiger stationär versorgt werden müssten. Die Klinik gerate hier in einen Zielkonflikt, da jene Patienten das RPB verstärkt belasteten:

„Wenn jemand aus einem anderen Landkreis kommt, übernimmt der Herkunftskreis die Finanzierung, d.h. wir haben inhaltlich keinen Einfluss mehr [auf die Leistung]." (GPZ_7)

"Wenn diese Patienten hier behandelt werden müssen, dann gehen sie auf das regionale Psychiatriebudget und das wird ein Problem für die Klinik." (EGH_1)

Aus Sicht der EGH stellt das Kooperationsziel „Ambulantisierung“ besonders in ländlichen Regionen eine Herausforderung dar. Die Hilfen seien vor allem in der Kreisstadt konzentriert, während periphere Ortschaften nur unzureichend über das öffentliche Verkehrsnetz angebunden seien. Ohne angemessen ausgebaute ambulante Versorgungsstrukturen blieben Instrumente zur Fallsteuerung weitgehend wirkungslos, kritisieren die Case-Manager: 
„Die EGH kann ambulant aufsuchend arbeiten und die Klinik bietet Home-Treatment und Krisendienst [...], aber es ist eben alles auf Büsum und Heide konzentriert und dazwischen ist wenig und die Leute können nicht kommen, die Busverbindungen sind schwierig."(EGH_2)

"Dass viele niederschwellige, ambulante Einrichtungen fehlen und deswegen das vollstationäre Angebot gar nicht auf einen anderen Bedarf umgestellt werden kann." (EGH_1)

\section{Diskussion}

Ein zentraler Aspekt der Ergebnisse betrifft die Einführung von Vergütungsmodellen, die ähnliche Steuerungsanreize setzen, bzw. die mit den Versorgungszielen der beteiligten Akteure in Einklang stehen. Dies deckt sich mit der Forschungsliteratur, wird dort sogar eine gemeinsame Finanzierung der Kooperationsbestandteile empfohlen [7,8]. Dass dies nicht nur zentrale Ingredienz, sondern auch limitierendes Moment der Zusammenarbeit ist, zeigt ein vergleichbares Projekt in Bremerhaven [17]: Dort wurde der Versuch unternommen, eine Managementgesellschaft zu gründen, die alle psychiatrischen Leistungen außerhalb der Regelversorgung steuern und in Form von multiprofessionellen gemeindepsychiatrischen Teams bündeln sollte. Die Finanzierung sollte über ein anbieterübergreifendes Budget geregelt werden. In der Umsetzung scheiterte das Projekt u.a. an den unterschiedlichen Vorstellungen der Akteure bzgl. Systemsteuerung und -vergütung [17].

In der vorliegenden Region wurde auf ein gemeinsames Budget verzichtet. Stattdessen vereinbarten Klinik und EGH gesonderte Budgets, die eine gleichbleibende Vergütung unabhängig vom jeweiligen Unterstützungssetting sicherstellen: Ob ein Klient im Heimbereich oder betreuten Einzelwohnen lebt oder eine Krisenintervention stationär oder aufsuchend erbracht wird, hat jeweils keine Auswirkung auf die Erlöshöhe. In der Literatur wird dieser Aspekt als Entkoppelung von Unterstützungsdichte (personelle Intensität einer Maßnahme) und Unterstützungssetting beschrieben [28]. In der Modellregion war dies nur begrenzt realisierbar, v.a. bedingt durch einen relativen Mangel ambulanter Strukturen der EGH [29].

\section{Maßnahmen zur Kooperation}

Ein Großteil der benannten Kooperationsmaßnahmen findet sich auch in der Forschungsliteratur. So sind die von Whiteford et al. [7] dargestellten Empfehlungen zur institutionsübergreifenden Vernetzung in der Modellregion bzw. vom GPZ mehrheitlich erfüllt (Tab. 2). Nicht umgesetzt wurden hingegen die gemeinsame räumliche Unterbringung der kooperierenden Dienste sowie ein gemeinsames Informationssystem. Diese Aspekte erfordern eine längerfristige vertragliche Regelung von Investitionskosten und datenschutzrechtlichen Fragen [7,8]. In der Untersuchungsregion betreiben daher sowohl EGH als auch Klinik eigene bauliche Strukturen und getrennte Informationssysteme. 
Tab. 2 Maßnahmen zur institutionsübergreifenden Vernetzung auf Systemebene (n. Whiteford et al. [7]) und Implementierung i.R. des GPZ

\begin{tabular}{|l|l|}
\hline Maßnahme & $\begin{array}{l}\text { Umsetzung } \\
\text { i.R. des GPZ }\end{array}$ \\
\hline Gemeinsame Angebotsplanung der kooperierenden Institutionen & $\mathrm{Ja}$ \\
\hline Steuerungsgremium zur übergreifenden Fallkoordination & $\mathrm{Ja}$ \\
\hline Einsatz eines die Zusammenarbeit koordinierenden Mitarbeiters & $\mathrm{Ja}$ \\
\hline Schriftliche Vereinbarung der Kooperationsbedingungen und -ziele & $\mathrm{Ja}$ \\
\hline Ähnliche Vergütungsformen zwischen den kooperierenden Institutionen & $\mathrm{Ja}$ \\
\hline $\begin{array}{l}\text { Gemeinsame Leistungserbringung durch interdisziplinäre und } \\
\text { institutionsübergreifende Teams }\end{array}$ & $\mathrm{Nein}$ \\
\hline $\begin{array}{l}\text { Gemeinsame Mitarbeitertrainings zur Förderung von gegenseitigem Verständnis } \\
\text { und einer kooperativen Haltung }\end{array}$ & $\mathrm{Ja}$ \\
\hline Gemeinsame räumliche Unterbringung & $\mathrm{Nein}$ \\
\hline $\begin{array}{l}\text { Nutzung eines gemeinsamen Informationssystems für das Fall- und } \\
\text { Datenmanagement }\end{array}$ & Nein \\
\hline
\end{tabular}

Der Einsatz (leitender) Mitarbeiter in Personalunion ist ein Aspekt, der sich auch in anderen Versorgungsmodellen bewährt hat, z.B. beim Berliner Krisendienst [30]. Ziel dieser verhältnismäßig leicht zu implementierenden Maßnahme ist es, bei den beteiligten Akteuren ein gegenseitiges (Netzwerk-) Wissen herzustellen. Ungewitter et al. [15] kritisieren hingegen die Anwendung eines die Zusammenarbeit koordinierenden Teammitglieds $[15,16]$. Dies mache Kooperationsbeziehungen störanfällig, etwa wenn entsprechende Personen ausfielen. Stattdessen schlagen sie regelmäßige Kooperationstreffen vor, zur Netzwerkpflege und gezielter Vermittlung von Netzwerkwissen.

\section{Implementierung des Modells}

In der Studienregion wurden eine Reihe struktureller und prozessualer Veränderungen vorgenommen (Abb. 1), um psychosoziale Leistungen stärker zu bündeln und in das häusliche Umfeld zu verlagern. Ein GPZ fungiert dabei als zentrale steuernde Instanz. Insgesamt wurde ein Großteil der Bestandteile des von Steinhart und Wienberg [18] konzipierten Funktionalen Basismodells für die gemeindepsychiatrische Versorgung implementiert (vgl. hierzu auch [24]). Zur Realisierung dieser Veränderungen waren in der Modellregion jedoch umfassende Verhandlungen zwischen der Klinik sowie den Leistungserbringern und Kostenträgern der EGH notwendig. Die Hürde, die vorbeschriebenen Innovationen auch in anderen Regionen zu implementieren, ist somit verhältnismäßig hoch. Hierzu fügen sich die Ergebnisse von Auschra [8], wonach zunächst die politischen und rechtlichen Grundlagen für interorganisationale Zusammenarbeit geebnet werden müssen.

\section{Limitationen}

Bedingt durch die strukturellen Besonderheiten, die im Zusammenhang mit dem GPZ in der Untersuchungsregion geschaffen wurden (z.B. die pauschale Budgetierung der Leistungen), sind die Ergebnisse nur eingeschränkt, allenfalls auf ländliche Versorgungskontexte, übertragbar. Unter den Studienteilnehmern (vgl. Tab. 2) fanden sich keine Akteure aus dem KV-Bereich, da diese zum Zeitpunkt der Erhebung nicht unmittelbar an der Zusammenarbeit im GPZ beteiligt waren. Trotz des modellhaften Charakters des Versorgungsangebotes stellt das GPZ einen wegweisenden Versuch dar, die psychosoziale Basisversorgung künftig ein 
Stück bedürfnisgerechter zu gestalten. Dafür sprechen auch die positiven Erfahrungen der Nutzer mit diesem Angebot [24].

\section{Konsequenzen für Klinik und Praxis}

Die Implementierung einer zentralen Beratungs- und Koordinationsfunktion für psychiatrische und Teilhabeleistungen ermöglicht passgenauere Hilfen und stärkt die Zusammenarbeit der Stakeholder in einer Region. Einheitliche Vergütungsmodelle zwischen den beteiligten Anbietern setzen Anreize für eine gemeinsame Ausrichtung der Versorgung. Zur Förderung von Ambulantisierung und Flexibilisierung stellen pauschale Budgets (z.B. §64b SGB V) eine geeignete Finanzierungsform dar.

\section{Förderung.}

Die Studie wurde vom Land Schleswig-Holstein gefördert (Az. 234 - 455.5.004-005/02).

\section{Einhaltung ethischer Richtlinien}

Interessenskonflikt. Die Autoren geben an, dass kein Interessenskonflikt besteht. 


\section{Literatur}

${ }^{1}$ Deister A, Zeichner D, Witt T, Forster H-J. Veränderung der psychiatrischen Versorgung durch ein Regionales Budget. Psychiatr Prax 2010; 37: 335-342

${ }^{2}$ Schmid P, Steinert T, Borbé R. Systematische Literaturübersicht zur Implementierung der sektorübergreifenden Versorgung in Deutschland. Psychiatr Prax 2013; 40: 414-424

${ }^{3}$ Greve N. Annäherungen an eine gemeindepsychiatrische Basisversorgung. Psychiatr Prax 2018; 45: 285-287

${ }^{4}$ Salize HJ, Lambert M. Flexibilisierung der Sektorgrenzen? Pro \& Kontra. Nervenarzt 2016; 87: 315318

${ }^{5}$ Klever-Deichert G, Rau F, Tilgen M. Das PsychVVG in der Gesamtschau. Krankenh 2017; 109: 98-109

${ }^{6}$ Diminic S, Carstensen G, Harris MG, Reavley N, Pirkis J, Meurk C, Wong I, Bassilios B, Whiteford HA. Intersectoral policy for severe and persistent mental illness: review of approaches in a sample of high-income countries. Glob Ment Health 2015; 2, e18

${ }^{7}$ Whiteford H, McKeon G, Harris M, Diminic S, Siskind D, Scheurer R. System-level intersectoral linkages between the mental health and non-clinical support sectors: a qualitative systematic review. Aust N Z J Psychiatry 2014; 48: 895-906

${ }^{8}$ Auschra C. Barriers to the Integration of Care in Inter-Organisational Settings: A Literature Review. Int J Integr Care 2018; 18, 1

${ }^{9}$ Kvarnström S. Difficulties in collaboration: a critical incident study of interprofessional healthcare teamwork. J Interprof Care 2008; 22: 191-203

${ }^{10}$ Magnusson A, Lützén K. Factors that influence collaboration between psychiatric care and CSSs: experiences of working together in the interest of persons with long-term mental illness living in the community. Scand J Caring Sci 2009; 23: 140-145

${ }^{11}$ Elstad TA, Eide AH. User participation in community mental health services: exploring the experiences of users and professionals. Scand J Caring Sci 2009; 23: 674-681

${ }^{12}$ Andvig E, Syse J, Severinsson E. Interprofessional Collaboration in the Mental Health Services in Norway. Nurs Res Pract 2014; 2014: 1-8

${ }^{13}$ Smith-Merry J, Gillespie J, Hancock N, Yen I. Doing mental health care integration: a qualitative study of a new work role. Int J Ment Health Syst 2015; 9: 32

${ }^{14}$ Stergiopoulos V, Saab D, Francombe Pridham K, Aery A, Nakhost A. Building flexibility and managing complexity in community mental health: lessons learned in a large urban centre. BMC Psychiatry 2018; 18: 20

${ }^{15}$ Ungewitter C, Böttger D, El-Jurdi J, Kilian R, Losert C, Ludwig K, Steinkohl V, Bramesfeld A. Struktur und Kooperation in der Versorgung psychisch Kranker. Nervenarzt 2013; 84: 307-314

${ }^{16}$ Ruppert D, Stegbauer C, Bramesfeld A, Bestmann B, Szecsenyi J, Götz K. „Die Hoffnung stirbt zuletzt ..." - sektorenübergreifende Kooperation in der Integrierten Versorgung. Psychiatr Prax 2017; 44: $134-140$ 
${ }^{17}$ Peters U. Erfolgreich scheitern - Die Folgen eines Projekts zur Einführung stationsersetzender Behandlung und ihrer Steuerung. Gesundheitswesen 2018; 80: 65-69

${ }^{18}$ Steinhart I, Wienberg G. Das Funktionale Basismodell für die gemeindepsychiatrische Versorgung schwer psychisch kranker Menschen - Mindeststandard für Behandlung und Teilhabe. Psychiatr Prax 2016; 43: 65-68

${ }^{19}$ Petersen H-P, Hejnal T. Regionales Psychiatriebudget. Psych Pflege Heute 2010; 16: 40-43

${ }^{20}$ Schröder B, Fleßa S. Regionalbudgets in der Psychiatrie: Eine Alternative zu tagesgleichen Pflegesätzen und zum zukünftigen Finanzierungssystem? Psychiatr Prax 2017; 44: 446-452

${ }^{21}$ von Peter S, Ignatyev Y, Indefrey S, Johne J, Schwarz J, Timm J, Heinze M. Spezifische Merkmale zur Einstufung der Modellversorgung nach § 64b SGB V. Nervenarzt 2018; 89:559-564

${ }^{22}$ Berghöfer A, Hense S, Birker T, Hejnal T, Röwenstrunk F, Albrecht M, Erdmann D, Stöckigt B. Costeffectiveness of a Coordination Model in Psychosocial Care. Integration of Health Care and Social Rehabilitation. Process 2018;

${ }^{23}$ Ostermann J, Erdmann D, Birker T, Hejnal T, Albrecht M, Röwenstrunk F, Stöckigt B, Berghöfer A. Cost evaluation of an innovative outreach clinic across Social Insurance Codes in rural northern Germany. Health Soc Care Community. 2019;00:1-11.

${ }^{24}$ Schwarz J, Berghöfer A, Brückner B, Birker T, Stöckigt B. Psychosoziale Beratung in einem gemeindepsychiatrischen Zentrum - eine qualitative Studie über die Erfahrungen der Nutzer. PPmP - Psychother Psych Med 2018; 1-18

${ }^{25}$ Witzel A. The Problem-centered Interview. Forum Qual Sozialforschung Forum Qual Soc Res 2000; 1 Im Internet: http://www.qualitative-research.net/index.php/fqs/article/view/1132

${ }^{26}$ Mayring P. Qualitative Inhaltsanalyse: Grundlagen und Techniken. 12. Aufl. Weinheim, Bergstr: Beltz, 2015

${ }^{27}$ Strauss A, Corbin JM. Grounded theory in practice. Thousand Oaks: Sage, 1997

${ }^{28}$ Wienberg G. 40 Jahre Psychiatriereform in Deutschland - Auf dem Weg in die Drei-KlassenPsychiatrie?! Sozialpsychiatrische Informationen 2014; 44: 4-9

${ }^{29}$ Gesundheitsministerium Schleswig-Holstein. Ausgabenanstieg der Eingliederungshilfe für Menschen mit Behinderung. Im Internet: http://www.landesrechnungshofsh.de/file/bemerkungen_2012_tz31.pdf

${ }^{30}$ Bergold JB, Zimmermann R-B. Wie arbeitet ein Krisendienst? PiD - Psychother Im Dialog 2003; 4: 382-388

${ }^{31}$ Fisher MP, Elnitsky C. Health and Social Services Integration: A Review of Concepts and Models. Soc Work Public Health 2012; 27: 441-468 


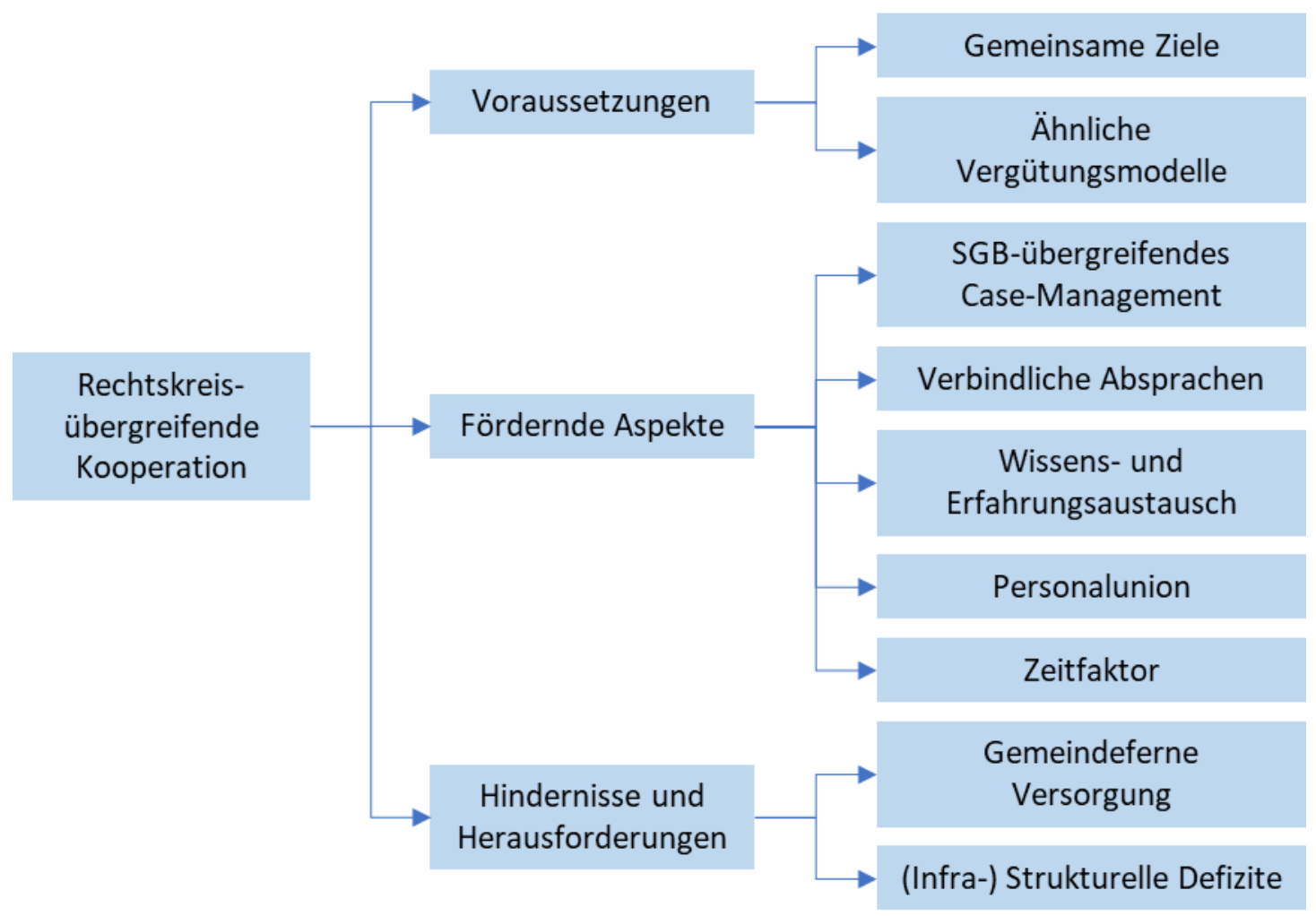

Abb. 2 Rechtskreisübergreifende Kooperation - Haupt- und Unterkategorien im Überblick. 